\title{
ANALYSIS OF LOGIC AND STRATEGY GAMES AS A TOOL FOR THE TEACHING AND APPROACH TO MATHEMATICAL CONCEPTS
}

\author{
S. Lantarón', M. López¹, S. Merchán ${ }^{1}$, J. Rodrigo² \\ ${ }^{1}$ Universidad Politécnica de Madrid (SPAIN) \\ ${ }^{2}$ Universidad Pontificia Comillas (SPAIN)
}

\begin{abstract}
The study presented in this paper shows an original and exhaustive analysis of some logic and strategy games. This kind of games represents a very useful tool for the teaching and a good approach to some mathematical concepts that must be treated in the different levels of the mathematical courses.

This work is a personal view of the authors that allows the use of some games as a way to learn mathematics. It is the result of years of experience in gamification field performed in the Aula Taller Museo de las Matemáticas m-ensa (https://innovacioneducativa.upm.es/museomatematicas/). While working with students of all levels we have realized that this kind of games is a perfect instrument for the teaching and learning of mathematics.

Our proposal involves strategy and logic games created by us as well as commercial ones. The main goal is to use the didactical value of them to introduce and consolidate some topics that the students must handle related to mathematical thinking.
\end{abstract}

We want to offer students the opportunity of playing and overcoming challenges as a way to reinforce the acquired learning during the current classes as well as to train their capacity of reasoning.

The analysis that we have done of each game, offers to professors the opportunity of using them as a motivating tool to introduce mathematical concepts.

Keywords: logic games, serious games, mathematical teaching methodologies, gamification, innovation.

\section{INTRODUCTION}

The authors of this study are members of the technical and management team of the Aula Taller-Museo de las Matemáticas $\pi$-ensa. This project is part of the "Pensamiento Matemático" Educational Innovation Group of the Universidad Politécnica de Madrid (UPM) which opened in September of 2014. The creation of a Mathematics workshop/museum hall was intended to convert the space into a cultural centre for the education community and the general public, offering distinct activities related to mathematics. Its general approach revolves around games of logic and strategy.

A good game, having well-defined rules and a rich logic content, requires certain mathematical concepts and analyses, similar to those necessary for problem solving, typical of this science.

Mathematics is, to a great extent, game playing and often, this game play can be analysed using mathematical instruments. In games, the goal is entertainment, quickly entering into action and competitiveness. This helps promote an interest in mathematics classes and topics while also fostering knowledge of the studied concepts. In this work, stimuli and motivations are used in order to instil the spirit of the game in the students, while at the same time, introducing and reinforcing specific topics that form a part of the course curriculum in distinct levels of learning (from primary school to university).

Some of the questions arising in this area include: Is it really possible to use games to improve mathematics education? How can this be done? What games? What objectives can be achieved through these games? This work responds to these questions through an in-depth analysis of some of the diverse logic and strategy games that have been created by the authors of the article and others available at different recreational and educational stores.

The authors support the gamification in mathematics. Our experience allows us to affirm that it is necessary to include new methodological tools that are appealing to students. We believe in the need to find alternative ways of educating, emphasizing essential individual skills, promoting creativity, personal initiative and self-learning and integrating educational innovations and initiatives with games playing a major role. This work is framed within these lines: game play as an ideal instrument for learning. In this case, focusing on students of distinct ages. 
The proposal specifically considers logic and strategy games and the objective is to use the didactic value of these games for the presentation and consolidation of the knowledge of certain mathematics topics that students should know, and for skills related to logical-mathematical thinking. Students should be offered the opportunity to play and overcome challenges, thus this mathematical knowledge will be of great assistance.

In most games, participants play to win, to overcome the challenges proposed by the game. And to win in the games that are presented, it is necessary to resort to mathematics-based skills that students should already know or are learning in their classes. Students are also required to observe game play, count, make deductions, generalize results, plan future moves, investigate potential new methods or strategies, all necessary elements for their academic education.

In this way, with this proposal, professionals and students are given the opportunity to use certain logic games in order to work on what they are learning in their daily classroom studies, as well as to train their powers of reasoning.

\section{METHODOLOGY}

Without a doubt, mathematics is one of the didactic fields in the most need of advances. While other scientific disciplines have recently revealed great didactic progress, in mathematics classes, this progress is occurring at a much slower pace. On many occasions, it continues to be unrelated to the daily reality thereby converting it into a set of mechanical and unconnected exercises. So, we feel that it is important to develop new tools or to adopt the already existing ones that are useful and easy to implement in the classroom, always in relation to the curriculum.

We have developed diverse educational and innovative tools through logic games to permit the understanding and application of distinct mathematical concepts and theories, through manipulation.

Methodology developed:

- Search for existing logic and strategy games available on the market that may be proposed from a mathematical perspective.

- Creation of new games in which the use of mathematical concepts and results will be of great use.

- Propose that students solve the challenges proposed by the distinct games. Guide them in their resolution through mathematical approaches.

\subsection{How to work with the students}

The work that we propose with the students is carried out as follows:

Proposing a session of "Games in the Classroom", in which different games are played by the students. Initially, they are given time to overcome the challenges, analysing the reasoning used.

Then, a "mathematical" strategy is proposed for the resolution of each of these challenges. The mathematical content proposed by these strategies is explained and discussed and the students are allowed to analyse and consider these.

The final activity explained in each game as well as the diverse mathematical concepts and results permit the resolution of the same, while also showing students how to use them, makes this resolution much more simple than working without a specific methodology.

\subsection{Analysis of some of the developed proposals}

To serve as a sample, some of the games that we used are presented below, along with their mathematical analysis.

\subsubsection{Balance Beans. Physics and geometry all in one}

In this case, a commercial game available on the market and that can assist with numerous mathematical concepts has been proposed in "Fig 1". It permits the player to quickly overcome the distinct challenges proposed. 


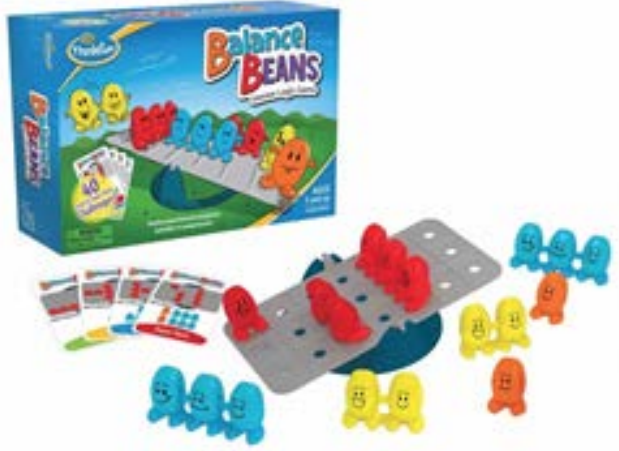

Figure 1. Balance Beans, a game of strategy with numerous didactic applications.

Balance Beans is a game of logic in which distinct challenges are presented on cards. First, the red beans should be placed as indicated on each challenge card (these beans cannot be moved). Then, all the other coloured beans should be placed on the seesaw, as indicated on the card in order to maintain its balance.

Some authors have proposed overcoming the challenges of this game using equations. That is:

The idea is to place the origin and initial value 0 in the centre of the seesaw (site where the platform is supported) and the rest of the positions (rows where the beans are situated on both sides) are numbered according to the distance of this row to the initial value 0 represented in "Fig 2".

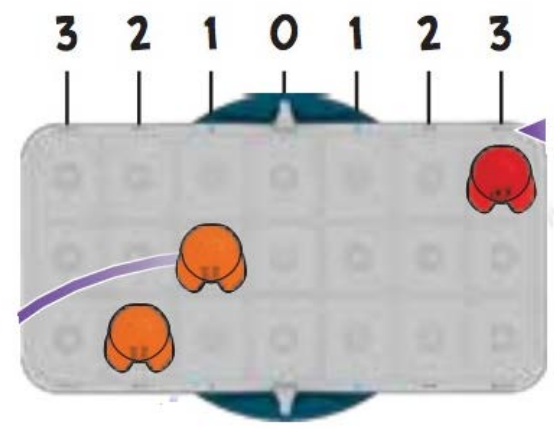

Figure 2. Measurement strategy in Balance Beans.

We assume that this challenge is based on the initial position of "Fig $3(\mathrm{a})$ " and that it should be used to balance two individual blocks of three blue beans and two orange beans. How do we place them to ensure the balancing without using the tedious trial and error method?

Multiply the value assigned to each row by the number of beans situated in it "Fig 3 (b and c)". The solving of the challenge is based on the idea that the sum of the results obtained from both sides of the established origin are equal.

This is the equation to be solved. In this example, both sides will have the value of 10 "Fig 3 (d)". Solve the equation, solve the challenge.

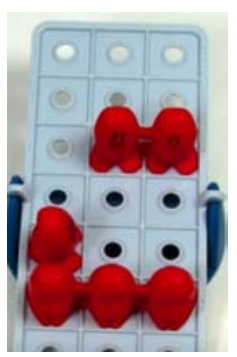

(a)

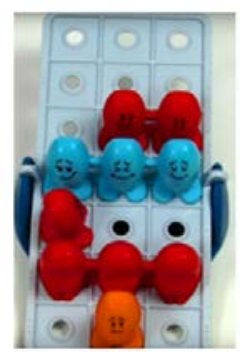

(b)

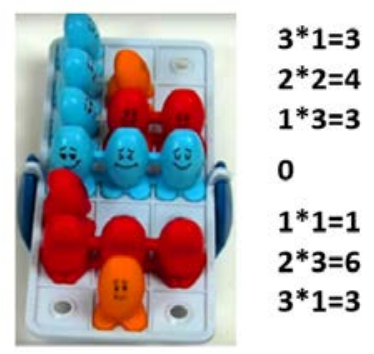

(c)

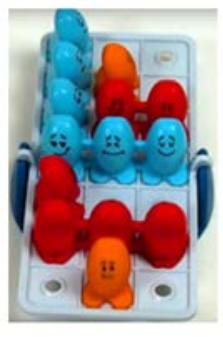

$3 * 1=3$

$2 * 2=4 \quad 3+4+3=10$

$1 * 3=3$

$1 * 1=1$

$2 * 3=6 \quad 1+6+3=10$

3*1=3

(d)

Figure 3. Strategy to be followed, based on equations to solve the Balance Beans game. 
Based on this idea, our proposal is to consider this game through geometric concepts (areas of triangles and/or rectangles). This allows us to strengthen and apply this area of mathematics.

For this new approach, we consider some axes of coordinates that are situated in the seesaw according to "Fig 4". On the vertical axis the number of beans in one row and in the horizontal axis, the value of the row:

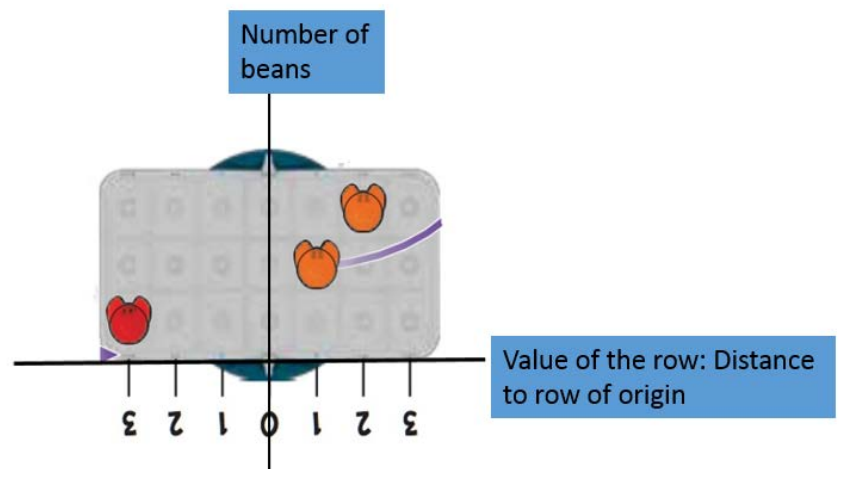

Figure 4. Consideration of axes in the seesaw.

The answer to each challenge is explained with a specific example. Take the case of the challenge proposed in "Fig $5(\mathrm{a})$ ", whose solution is presented in "Fig 5 (b)":

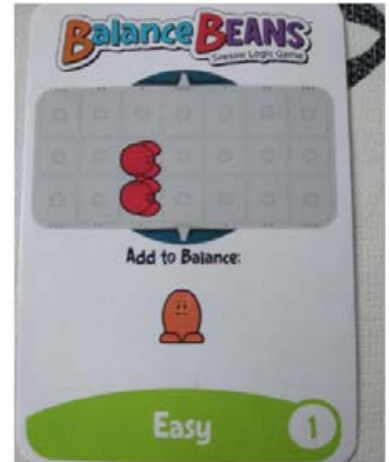

(a)

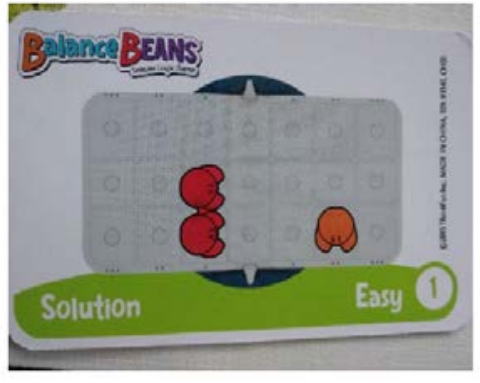

(b)

Figure 5: Challenge to solve and solution of the same.

Based on this, we make the geometric representation of "Fig 6", considering triangles with bases and heights corresponding to the previously mentioned axes: A triangle of base 1 and height 2 (two beans in a row of value 1) on one of the sides and another of base 2 and height 1 (a bean in the row of value 2 ) on the other side. As can be seen, solving the challenge is the same as searching for the sum of the areas of the triangles that coincide on both side of the vertical axis ("Fig 7").

For each challenge, the student, in placing the beans, should seek this balance of sums of the areas.

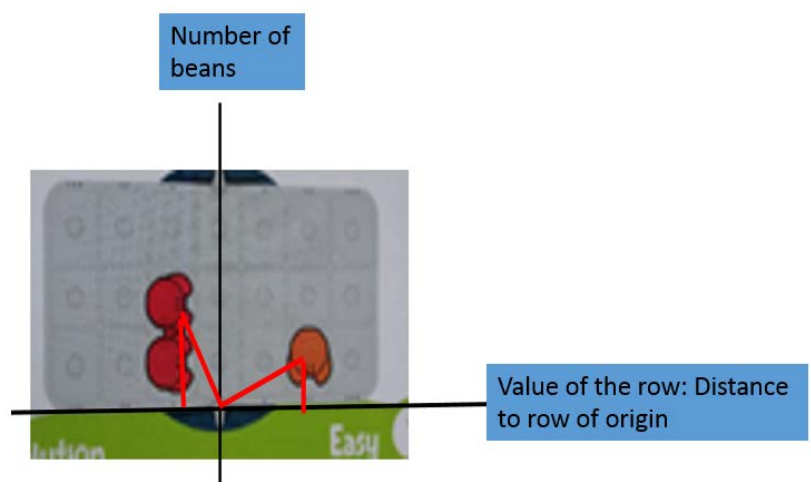

Figure 6: Geometric approach to the problem considering triangles. 


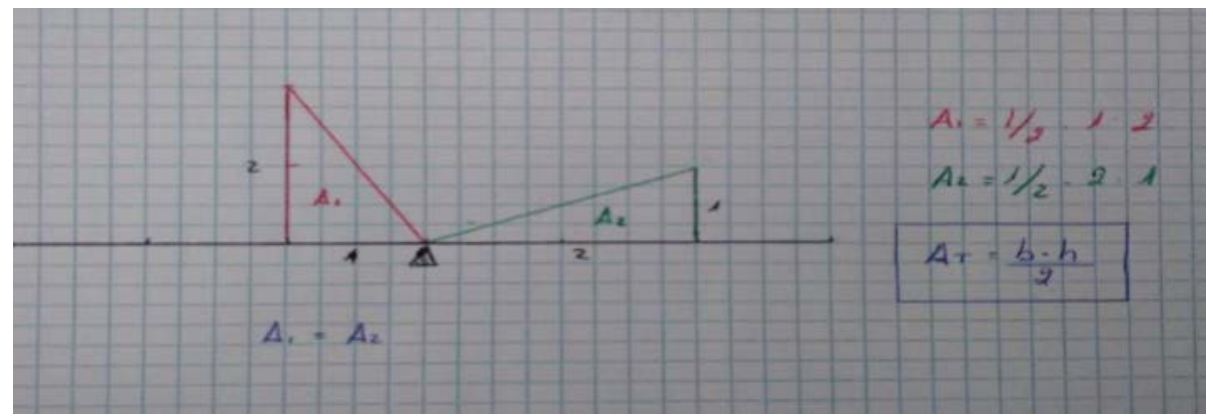

Figure 7: Problem solving according to triangle areas.

Similarly, the problem may be approached by considering rectangles instead of triangles, with the measurements of their sides being equal to the number of beans in the row and the value of the row.

\subsubsection{A classic puzzle. Geometric solution}

This simple game, very easy to create, permits the use of diverse geometric concepts in an applied and appealing manner. Normally, this type of challenge is solved by applying the Pythagorean Theorem. The proposed strategy is based on this idea, considering the geometric approach without the need to carry out any type of operation.

It is a well-known puzzle made up of 5 pieces in which two consecutive challenges are proposed ("Fig 8"):

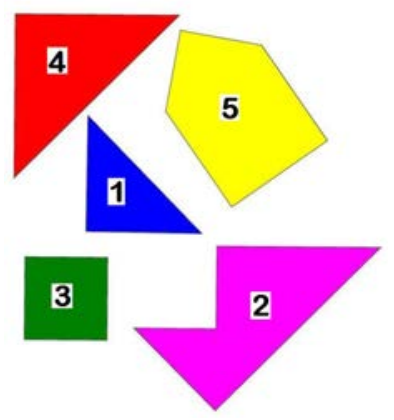

Figure 8. Square puzzle.

$1^{\text {st }}$.- Removing the square piece (piece number 3 ), with the four remaining pieces, you should construct a square. Once managing to do this, a more difficult challenge is proposed:

$2^{\text {nd }}$.- With all of the pieces, construct another square.

Solving the first challenge is quite simple ("Fig 9").

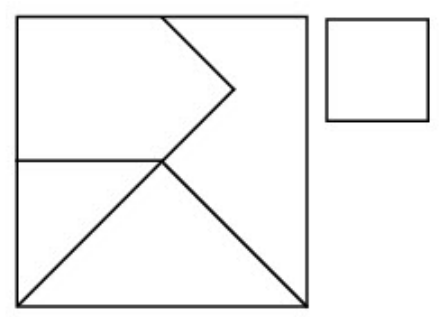

Figure 9. Solution of the first challenge of the square puzzle.

The second proposal is more complicated, focusing on solving through trial and error, which may be quite tedious and frustrating. It is convenient to first carry out a mathematical study that will help to determine the appropriate path.

Referring to the first square that was created in the initial challenge as $\mathrm{C} 1$, and with $\mathrm{A} 1$ being its area, and with $\mathrm{C} 2$ and $A 2$ being the square that should be added and its area (piece 3 ), the new square $C$ 
that is to be created should have an area of $A 1+A 2$. It is sufficient to apply the Pythagorean Theorem to the right triangle, whose legs are the sides of squares $\mathrm{C} 1$ and $\mathrm{C} 2$, to determine the side of the square that should be constructed ("Fig 10").

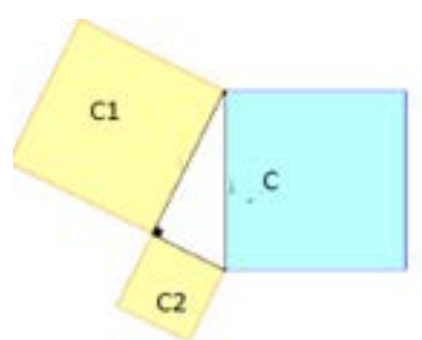

Figure 10. Pythagorean Theorem for the geometric approach to solve the second challenge.

Our proposal is based, therefore, on the idea that, given the prior approach, determining the side of the square that is being sought out, considering the hypotenuse of the right triangle, a good way to solve the challenge would be to place the squares C1 and C2 as appears in "Fig 11" and continue to seek the combination of pieces that achieves the side of square $C$ (the hypotenuse). Once found, complete the desired square.

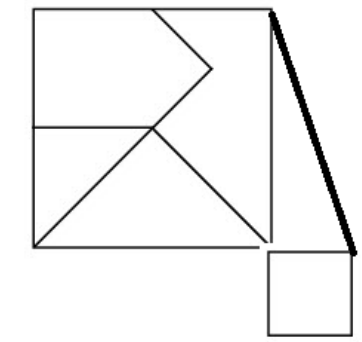

Figure 11: Solving the $2^{\text {nd }}$ challenge without the need to carry out operations

\subsubsection{Other proposals}

Like those proposed in the previous sub-sections, other games have been invented and classic games have been adapted in order to work with other mathematical concepts. These include:

- Binary Guess Who? The proposal begins by explaining the moment in which mathematics serves as a support to computer science by using algorithms. To create them, it is necessary to understand the concept of Yes, No or, as referred to in mathematics, 0 and 1 (Binary System). The developed game helps with the understanding of this system, adapting the classic game of "Guess Who?" In this game, it is necessary to make up questions to be responded to with a yes or no answer, in order to find out the most information-- just like the creation of an algorithm.

- Functional Cluedo. In this case, the adaptation of Cluedo has been created to determine which function has disappeared, why and under what circumstances. This promotes the knowledge of all of the real functions of a real variable.

\section{RESULTS AND CONCLUSIONS}

The implementation of this proposal with diverse student groups has achieved the proposed objectives, which can be summarized as follows:

- Awakening an interest in the learning of mathematics.

- Ensuring that students relate mathematics with real life and know how to apply the acquired knowledge.

- Strengthening skills that are based on mathematical reasoning: strategy, planning, decisionmaking, etc. 
- Applying the dynamics and principles of games to improve motivation, interest and implication of students in areas of mathematical content.

- To create a series of recreational proposals (games) to strengthen mathematical knowledge and the enjoyment of this science.

Achieving these objectives means facilitating the student's learning process, using a pleasant, flexible, dynamic and interactive approach, which attempts to attract students and promote their implication in the subject.

We are convinced that the actions proposed in this project will contribute to an increased involvement of the students in the basic areas that are necessary for their studies. The application of games is very motivating and is a good means of reinforcement if applied to mathematical topics. Furthermore, this permits students to integrate and relate amongst one another, given that team actions are proposed.

The class groups that were presented with the activity revealed an excellent understanding of the presented content and improved their perception of the topics at hand.

Future actions should include student implication in the co-design or invention of new proposals (new games) so that they may use the content that they have learned or are learning in the classes related to mathematics. This would help to stimulate their creativity and mathematical knowledge.

\section{ACKNOWLEDGEMENTS}

This work has been partially financed by the Educational Innovation Project E-Pi-LOG-0, which is part of the 2018-19 convocation for Support for Educational Innovation and Improvements in Teaching Quality at the Universidad Politécnica de Madrid.

\section{REFERENCES}

[1] R. Araya, P. Dartnell, C. Aguirre, E. Tiedemann, M.J. Contreras, M. Araya, and P. Calfucura, "Saber pedagógico y conocimiento de la disciplina matemática en profesores de educación general básica”. Primer concurso FONIDE, 2008. <https://centroestudios.mineduc.cl/wpcontent/uploads/sites/100/2017/07/2006-UChile-Araya-1.pdf>.

[2] C.E. Arteaga, "El desarrollo de la creatividad en la Educación Matemática". Congreso Iberoamericano de Educación, Metas 2021, Buenos Aires, 2010.

[3] R. Brown, "Teorías matemáticas creadoras e imaginativas", Blume, 50, 2012.

[4] M. De Guzmán, Para pensar mejor: Desarrollo de la creatividad a través de los procesos matemáticos. Madrid: Pirámide, 1995.

[5] K. Robinson, "How Schools Kill Creativity". TED, Monterey, California, 2006.

[6] J. Tanton, "Solve This: Math Activities for Students and Clubs", Mathematical Association of America, United States of America, 2001. 